\title{
Excited States for Coulomb Systems in the Hartree-Fock Approximation
}

\author{
Jean-François Leon*
}

Service de Physique Atomique des Plasmas, Centre d'études de LIMEIL BP 27, F-94190 VilleneuveSt Georges, France

\begin{abstract}
In this paper, we define excited states for Coulomb systems in the Hartree-Fock approximation as minima of the Hartree-Fock energy on a set of constraints depending on excited states of the lower energy. There are several ways to define such a procedure: we study four of them and prove an existence result for these excited states.
\end{abstract}

\section{Introduction}

We present here an existence result for a class of minimization problems related to excited states in Hartree-Fock (HF) theory. We consider the standard description of non-relativistic electrons in a coulombic field. The $N$-body Hamiltonian is then

with

$$
H=\sum_{i=1, N}\left(-\Delta_{x_{i}}+V\left(x_{i}\right)\right)+\sum_{1 \leqq i<j \leqq N} 1 /\left|x_{i}-x_{j}\right|
$$

$$
V(x)=-\sum_{j=1, m} z_{j} /\left|x-\underline{x}_{j}\right|,
$$

where $m \geqq 1$ is the number of nuclei and $z_{j}>0$ and $x_{j}$ are respectively charges and position of nuclei ( $x_{i}$ denotes the position of one particle in $R^{3}$ ). Furthermore, we denote by $Z=\sum_{j=1, m} z_{j}$ the total charge, where $N$ is the number of electrons.

We assume in this paper $Z \geqq N$. As usual, $H$ acts on $L^{2}{ }_{a}\left(R^{3 N}\right)$ (antisymmetric functions of $L^{2}\left(R^{3 N}\right)$. We neglect the spin dependence but everything we say below may be trivially adapted to spin dependent functions.

There is a functional called the "energy" which is related to $H$; it is classically defined as follows:

$$
\begin{array}{r}
E(\varphi)=\int_{R^{3 N}}\left(|\nabla \varphi|^{2}+\left(\sum_{i=1, N} V\left(x_{i}\right)+\sum_{1 \leqq i<j \leqq N} 1 /\left|x_{i}-x_{j}\right|\right)|\varphi|^{2}\right) d x=(\varphi, H \varphi) \\
\text { with } \varphi \in H^{1}{ }_{a}\left(R^{3 N}\right) .
\end{array}
$$

* Present address: Centre d'etudes de Gramat, F-46500 Gramat, France 
In other words, an energy is here the quadratic form associated with the self-adjoint operator $H$. The functional $E$ completely describes the system.

We notice that $\varphi \in L_{a}^{2}\left(R^{3 N}\right)$ is a priori complex-valued. Nevertheless, since we are interested in the spectrum of $H$ and since $H$ is real and self-adjoint, we can deal with real-valued functions.

Unfortunately, the study of $E$ on $L^{2}{ }_{a}\left(R^{3 N}\right)$ is hopeless for $N \geqq 2$ and approximations are needed. The so-called Hartree-Fock approximation is the usual approximation in atomic and molecular physics. See Cowan [1] for the practical use of this approximation in computational physics.

The basis of the method consists in restricting the study of $E$ to a subspace of $L_{a}^{2}\left(R^{3 N}\right)$ sufficiently large to provide good estimates but sufficiently tractable to allow numerical computations. This is the space of Slater determinant wavefunctions $S_{N}$, so that

$$
\Phi \in S_{N} \Leftrightarrow \Phi\left(x_{1}, x_{2}, \ldots, x_{N}\right)=(1 / \sqrt{(N !)}) \operatorname{det}\left(\varphi_{i}\left(x_{j}\right)\right)
$$

where

$$
\varphi_{i} \in H^{1}\left(R^{3}\right) \forall i \quad \text { and } \quad\left(\varphi_{i}, \varphi_{j}\right)=\delta_{i j} \forall i, j .
$$

Now $E$ restricted to $S_{N}$ may be written $E(\Phi)=\mathbb{E}\left(\varphi_{1}, \varphi_{2}, \ldots \varphi_{N}\right)$, where

$$
\begin{aligned}
\mathbb{E}\left(\varphi_{1}, \varphi_{2}, \ldots \varphi_{N}\right)= & \sum_{i=1, N} \int_{R^{3}}\left(\left|\nabla \varphi_{i}\right|^{2}+V(x)\left|\varphi_{i}\right|^{2}\right) d x+1 / 2 \\
& \cdot\left(\iint_{R^{3} \times R^{3}}\left(\left(\rho(x) \rho(y)-|\rho(x, y)|^{2}\right) /|x-y|\right) d x d y\right),
\end{aligned}
$$

where we employ the notations $\rho(x)=\sum_{i=1, N} \varphi_{i}(x)^{2}, \rho(x, y)=\sum_{i=1, N} \varphi_{i}(x) \varphi_{i}(y)$. (Recall we are dealing with real functions.)

This functional is defined on the set

$$
K=\left\{\left(\varphi_{i}\right) 1 \leqq i \leqq N ; \forall i, \varphi_{i} \in H^{1}\left(R^{3}\right),\left(\varphi_{i}, \varphi_{j}\right)=\delta_{i j} \forall i, j\right\} .
$$

The derivation of (3)-(4) from (2) may be found for instance in Lions [4].

Lieb-Simon [3] show the existence of a ground state of (3)-(4); Lions [4] gives various proofs of this fact and defines excited states as critical points of $\mathbb{E}$.

In this paper, we define excited states in another way as solutions of successive constrained minimization problems and we show the existence of such excited states. On the other hand, we define another class of minimization problems related to the sum of the $M^{\text {th }}$ first eigenvalues of $H$ and show an existence result for these problems. We follow the method proposed in Lions [4].

\section{Minimization Problems and Existence Results}

We consider four minimization problems, which consist in minimizing the functional $\mathbb{E}$ on various sets derived from $S_{N}$. The purpose of these minimization problems is to provide approximations for the energies of the excited states. The idea is that if we minimize the function $\mathbb{E}$ on the set of normalized Slater determinant wavefunctions which are orthogonal to the approximate ground state, 
we compute an approximation for the first excited state. Nevertheless, there are various ways to iterate the procedure. We study two of them, which we denote by $P_{1}(K, \theta)$ and $P_{2}(K, \theta)$. On the other hand, we remark that from a mathematical point of view, the determination of the $M^{\text {th }}$ first eigenvalues should be transformed into the determination of the successive sums of the $X^{\text {th }}$ first eigenvalues for every $X \leqq M$. This reformulation of $P_{1}(K, \theta)$ and $P_{2}(K, \theta)$ provides two other minimization problems, $P_{4}(K)$ and $P_{3}(K)$; but the connections between these problems and $P_{1}, P_{2}$ are nontrivial. Nevertheless, it is easy to see that the last two approximations are the best ones, but they are much more expensive if we want numerical results.

Let us explain in detail now how these different methods arise. Assume that we have determined $M-1$ Slater determinant wave functions, $\Phi_{i}=\left(\varphi_{i j}\right) 1 \leqq i \leqq$ $M-1,1 \leqq j \leqq N$. We want to determine a normalized Slater determinant wavefunction $\Phi$, orthogonal to the $M-1\left(\Phi_{i}\right)$. A tractable numerical method should transform this global orthogonality condition into an orthogonality condition on the one particle wavefunctions. It is this tractable constraint which induces the nonuniqueness of the procedure. Indeed two nonequivalent orthogonality conditions are:

1. In $P_{1}$, we assume that each single-particle wavefunction of the Slater determinant we are minimizing is orthogonal to one single-particle wavefunction of the $M-1$ Slater determinants already determined. So, we write for $P_{1}(K, \theta)$,

$$
P_{1}(K, \theta)\left\{\begin{array}{l}
\operatorname{Inf} \mathbb{E}\left(\varphi_{1}, \varphi_{2}, \ldots \varphi_{N}\right) \\
\left(\varphi_{1}, \varphi_{2}, \ldots, \varphi_{N}\right) \in K \\
\left(\theta_{l}, \varphi_{i}\right)=0 \text { for all } l, i
\end{array}\right.
$$

where $\theta=\left\{\theta_{l}\right\}$ is a given finite set of functions of $H^{1}\left(R^{3}\right)$

The $M^{\text {th }}$ Slater determinant is determined by considering the finite infimum

$$
\operatorname{Min}\left\{P_{1}(K, \theta) / \theta=\left\{\underline{\varphi}_{l j(l)}\right\} 1 \leqq l \leqq M-1,1 \leqq j(l) \leqq N\right\} .
$$

2. In $P_{2}$, we assume that one single-particle wavefunction of the Slater determinant we are minimizing is orthogonal to each single-particle wavefunctions of the $M-1$ Slater determinants already determined. Thus we write

$$
P_{2}(K, \underline{\theta})\left\{\begin{array}{l}
\operatorname{Inf} \mathbb{E}\left(\varphi_{1}, \varphi_{2}, \ldots \varphi_{N}\right) \\
\left(\varphi_{1}, \varphi_{2}, \ldots \varphi_{N}\right) \in K \\
\left(\underline{\theta}_{\rho}, \varphi_{1}\right)=0 \forall l \underline{\theta}_{\rho} \in H^{1}\left(R^{3}\right) \quad 1 \leqq l \leqq L=N(M-1) .
\end{array}\right.
$$

So the $M^{\text {th }}$ Slater determinant is determined by solving $P_{2}(K, \underline{\theta})$ with $\left\{\underline{\theta}_{l}\right\}=\left\{\varphi_{i j}\right\}$. Next, the reader should recognize in $P_{3}(M)$ defined below the minimization problem of giving an approximation of the sum of the $M^{\text {th }}$ first eigenvalues related to $P_{2}$, whereas $P_{4}$ is related to $P_{1}$,

$$
\begin{aligned}
& P_{3}(M)\left\{\begin{array}{l}
\mathbb{E}_{3}(M)=\operatorname{Inf}_{K_{M}} \sum_{i=1, M} \mathbb{E}\left(\varphi_{i 1}, \varphi_{i 2}, \ldots \varphi_{i N}\right) \\
K_{M}=\left\{\left(\varphi_{i j}\right)_{1 \leqq j \leqq N} \in K \forall i, 1 \leqq i \leqq M,\left(\varphi_{i_{1} 1}, \varphi_{i_{2} 2}\right)=0 \forall i_{1}, i_{2} / i_{1}>i_{2} \forall j\right\} .
\end{array}\right. \\
& P_{4}(M)\left\{\begin{array}{l}
\mathbb{E}_{4}(M)=\operatorname{Inf}_{K_{M}^{\prime}} \sum_{i=1, M} \mathbb{E}\left(\varphi_{i 1}, \varphi_{i 2}, \ldots \varphi_{i N}\right) \\
K_{M}^{\prime}=\left\{\left(\varphi_{i j}\right)_{1 \leqq J \leqq N} \in K \forall i, 1 \leqq i \leqq M,\left(\varphi_{i_{1} j}, \varphi_{i_{2} 1}\right)=0 \forall i_{1}, i_{2} i_{1}>i_{2} \forall j\right\} .
\end{array}\right.
\end{aligned}
$$


Let us emphasize here that $P_{3}$ and $P_{4}$ are distinct problems due to the fact that the constraint sets in these problems are distinct.

It is clear that from a numerical point of view, the formulation in terms of $P_{2}$ or $P_{1}$ is more tractable, the most efficient one being $P_{2}$. On the other hand the best results will be obtained with the formulation in terms of $P_{3}$ or $P_{4}$. Indeed we have the two inequalities that are easy to prove with the definitions of the constraint sets for the four problems:

$$
\begin{aligned}
& \mathbb{E}_{3}(M) \leqq \sum_{i=1, M} \mathbb{E}_{2}(i)<0, \\
& \mathbb{E}_{4}(M) \leqq \sum_{i=1, M} \mathbb{E}_{1}(i)<0 .
\end{aligned}
$$

The main result of our paper is an existence result for these problems.

Theorem 1. We assume $Z \geqq N$. Then every minimizing sequence of $P_{1}$ or $P_{2}$ is relatively compact in $H^{1}\left(R^{3}\right)^{N}$. Every minimizing sequence of $P_{3}$ or $P_{4}$ is relatively compact in $\left(H^{1}\left(R^{3}\right)\right)^{M N}$ and thus, each of these problems admits a minimum.

Remarks. 1) We use here without proof two properties of $\mathbb{E}$ which are proved in Lieb-Simon [3]. Namely, $\mathbb{E}$ is bounded from below on $K$ and, $\mathbb{E}$ is lower semi continuous on $H^{1}\left(R^{3}\right)$.

2) The physical situations that are enclosed in our result are neutral or positively charged systems.

\section{Proof of the Theorem}

Sketch of Proof. In the four cases, we follow the same method.

Step 1. A minimizing sequence for $P\left(=P_{1}, P_{2}, P_{3}\right.$ or $\left.P_{4}\right)$ is perturbed with a method due to Ekeland [2] in a way such that each element of the perturbed minimizing sequence should be seen as a minimum for a perturbed problem. Of course, the perturbed sequence is a minimizing sequence for $P$ again.

Step 2. Writing the non-negative Hessian condition for a perturbed problem, we deduce some bounds on the eigenvalues of the matrix of the Lagrange multipliers.

Step 3. Step 2 yields the compactness needed and the conclusion is then standard in view of the properties of $\mathbb{E}$ recalled above.

Before proving the theorem, we recall a general lemma. (See Lions [4] or the appendix for the proof.)

Lemma 1. Let $\mu$ be a bounded non-negative measure on $R^{3}$ such that $\mu\left(R^{3}\right)<Z$. Let $H_{1}$ be the Hamiltonian given by

$$
H_{1}=-\Delta+V+\mu * 1 /|x| .
$$

Then $H_{1}$ admits an increasing sequence of negative eigenvalues $\lambda_{n}$ converging to 0 as $n$ goes to $\infty$.

Finally, we detail the proof for $P_{2}$ only: the reader will convince himself that this proof may be easily adapted to the three other cases. 
Proof of the Theorem. Let $\left(\varphi^{n}{ }_{i}\right)_{1 \leqq l \leqq N}$ be a minimizing sequence for $P_{2}$. We use the general perturbation principle due to Ekeland [2], as in Lions [4], and we build another minimizing sequence $\left(\varphi^{n}{ }_{i}\right)_{1 \leqq l \leqq N}$ for $P_{2}$ such that $\left\|\varphi^{n}{ }_{i}-\varphi^{n}{ }_{i}\right\|_{L^{2}} \rightarrow 0$ as $n$ goes to $\infty$ and $\left(\varphi^{n}{ }_{i}\right)_{1 \leqq l \leqq N}$ is a minimum of the functional

$$
\mathbb{E}\left(\tau_{1}, \tau_{2}, \ldots \tau_{N}\right)+\mu_{n} \sum_{i=1, N}\left\|\tau_{i}-\varphi^{n}{ }_{i}\right\|_{L^{2}}
$$

with $\mu_{n}>0, \mu_{n} \rightarrow 0$ as $n$ goes to $\infty$. So we can write first order conditions and "non-negative Hessian" conditions verified by $\left(\varphi^{n}{ }_{i}\right)_{1 \leqq i \leqq N}$, minima of (5). These conditions may be written

$$
\begin{aligned}
& -\Delta \varphi^{n}{ }_{i}+V \varphi^{n}{ }_{i}+\left(\rho^{n} * 1 /|x|\right) \varphi^{n}{ }_{i}-\int_{R^{3}} \rho^{n}(x, y) \varphi^{n}{ }_{i}(y) /|x-y| d x d y+\left(A^{n} \varphi^{n}, e_{i}\right) \\
& \quad+\delta_{1 i}\left(\sum_{l=1, L} \alpha^{n}{ }_{l} \theta_{l}\right) \rightarrow 0 \quad \text { in } \quad L_{2}\left(R^{3}\right) \quad \forall i \quad 1 \leqq i \leqq N
\end{aligned}
$$

$\left(\delta_{1 i}=1\right.$ if $i=1,0$ in the other cases and $e_{i}$ is the vector with components $\left.\delta_{i j} 1 \leqq j \leqq N\right)$.

These equations are generalizations of standard HF equation. We recall the notations: $\rho^{n}(x, y)=\sum_{i=1, N} \varphi^{n}{ }_{i}(x) \varphi^{n}{ }_{i}(y)$ and $\rho^{n}(x)=\rho^{n}(x, x) \geqq 0$. $A^{n}$ is the symmetric matrix of Lagrange multipliers associated to the $n^{\text {th }}$ perturbed problems. We say that $\left(\varphi^{n}\right)_{1 \leqq i \leqq N}$ is a minimum for (5). Thus the Hessian of (5) is non-negative along an admissible direction. Indeed (after a lengthy but straightforward computation)

$$
\begin{aligned}
& \sum_{i=1, N} \int_{R^{3}}\left(\left|\nabla \chi_{i}\right|^{2}+V(x)\left|\chi_{i}\right|^{2}+\left(\rho^{n} * 1 /|x|\right)\left|\chi_{i}\right|^{2}\right) d x+\int_{R^{3}}\left(A^{n} \chi, \chi\right)+\mu_{n}(\chi, \chi) d x \\
& \left.\quad-\iint_{R^{3} \times R^{3}} \rho^{n}(x, y) \chi(x) \chi(y) /|x-y|\right) d x d y \\
& \quad+1 / 2 \iint_{R^{3} \times R^{3}}\left(\left|K^{n}(x, y)\right|^{2}-K^{n}(x) K^{n}(y)\right) /|x-y| d x d y \geqq 0,
\end{aligned}
$$

provided $\chi$ satisfies $\chi_{i} \in H^{1}\left(R^{3}\right)$ for every $i$ and

$$
\begin{array}{rl}
\left(\chi_{i}, \chi_{j}\right)=0 & i \neq j \\
\left(\chi_{i}, \varphi^{n}{ }_{j}\right)=0 & \forall i, j \\
\left(\underline{\theta}_{l}, \chi_{i}\right)=0 & \forall i, l,
\end{array}
$$

with the notations $K^{n}(x, y)=\sum_{i=1, N} \varphi^{n}{ }_{i}(x) \chi_{i}(y)+\varphi^{n}{ }_{i}(y) \chi_{i}(x)$ and $K^{n}(x)=K^{n}(x, x)$. Notice that the vector space on which the hessian of (5) may be negative has dimension at most NM. Let $e^{n}$ be the normalized eigenvector corresponding to $\varepsilon^{n}$, the smallest eigenvalue of $A^{n}$. Let $\chi=\theta e^{n}$ with $\left(\theta, \varphi^{n}{ }_{j}\right)=0 \forall j$ and $\left(\underline{\theta}_{l}, \theta\right)=0 \forall l$. Then we have as a special case of (7),

$$
\int_{R^{3}}\left\{\left(|\nabla \theta|^{2}+V(x)|\theta|^{2}+\left(\rho^{n} * 1 /|x|\right)|\theta|^{2}\right) d x+\int_{R^{3}} \varepsilon^{n}|\theta|^{2}+\mu_{n}|\theta|^{2}\right\} d x \geqq 0 .
$$

This inequality is deduced from (7) with $\chi=\theta e^{n}$, because we have

$$
\left.\iint_{R^{3} \times R^{3}} \rho^{n}(x, y) \chi(x) \chi(y) /|x-y|\right) d x d y \geqq 0
$$


and

$$
\iint_{R^{3} \times R^{3}}\left(\left(\left|K^{n}(x, y)\right|^{2}-K^{n}(x) K^{n}(y)\right) /|x-y|\right) d x d y \leqq 0 .
$$

The first inequality is standard $(1 /|x|$ is a positive type distribution), the second one is an application of the Cauchy-Schwarz inequality and is proved in Lions [4]. Now, assume extracting a subsequence if necessary, that $\lim \sup _{n}\left(-\left(\varepsilon^{n}+\mu_{n}\right)\right) \geqq$ 0 . We can assume without loss of generality that $\lim _{n}\left(-\left(\varepsilon^{n}+\mu_{n}\right)\right) \geqq 0$. On the other hand, the sequence $\rho^{n}(x)$ is strictly bounded in $L^{1}\left(R^{3}\right)$ by $N$, and for every $n$ the function $\rho^{n}(x)$ is nonnegative. So there is a non-negative measure $\rho$ such that $\rho^{n}$ converges toward $\rho$ (extracting a subsequence if necessary) in the weak topology of $M\left(R^{3}\right)$. Furthermore, $\rho\left(R^{3}\right)<N$. Then, passing to the limit in (8) provides the following estimate

$$
\int_{R^{3}}\left\{\left(|\nabla \theta|^{2}+V(x)|\theta|^{2}+(\rho * 1 /|x|)|\theta|^{2}\right) d x \geqq 0 .\right.
$$

Indeed, we have

$$
\int_{R^{3}}\left(\rho^{n} * 1 /|x|\right)|\theta|^{2} d x=\int_{R^{3}}\left(|\theta|^{2} * 1 /|x|\right) \rho^{n} d x
$$

and $|\theta|^{2} * 1 /|x|$ is in $L^{\infty}\left(R^{3}\right)$ for $\theta \in H^{1}\left(R^{3}\right)$. (Recall the well known inequality $|\theta|_{\infty} \leqq$ $C|\theta|_{2}|\nabla \theta|_{2}$.) From these facts, we deduce

$\lim \int_{R^{3}}\left(\rho^{n} * 1 /|x|\right)|\theta|^{2} d x=\int_{R^{3}}(\rho * 1 /|x|)|\theta|^{2} d x$ as $n$ goes to infinity.

Let $H_{1}$ be the operator defined by the formula

$$
H_{1}=-\Delta+V+\rho * 1 /|x| \text {. }
$$

The estimate (9) tells us that if $Z>N, H_{1}$ has at most a finite number of negative eigenvalues, an assertion which is in direct contradiction with Lemma 1. So

$$
\lim \sup \left(-\left(\varepsilon^{n}+\mu_{n}\right)\right)<0
$$

This yields for $n$ large enough the inequality

$$
\varepsilon^{n}+\mu_{n} \geqq \kappa>0 .
$$

Since $\mu_{n} \rightarrow 0$ as $n$ goes to infinity, we deduce that for $n$ large enough there exists an $\underline{\varepsilon}>0$ such that $\varepsilon^{n} \geqq \underline{\varepsilon}>0$, so $A^{n}$ is a positive matrix. Now, multiplying (6) by $\varphi^{n}{ }_{i}$ for each $i$ and summing on $i$ give the estimate

$$
\begin{aligned}
\operatorname{Tr}\left(A^{n}\right) \leqq & a_{n}-\left\{\sum_{i=1, N} \int_{R^{3}}\left(\left|\nabla \varphi^{n}{ }_{i}\right|^{2}+V(x)\left|\varphi^{n}{ }_{i}\right|^{2}\right) d x\right. \\
& \left.+\left(\int_{R^{3} \times R^{3}}\left(\left(\rho^{n}(x) \rho^{n}(y)-\left|\rho^{n}(x, y)\right|^{2}\right) /|x-y|\right) d x d y\right)\right\} \\
\leqq & a_{n}+\left|\mathbb{E}\left(\varphi^{n}{ }_{1}, \varphi^{n}{ }_{2}, \ldots \varphi^{n}{ }_{N}\right)\right|
\end{aligned}
$$

with $a_{n} \rightarrow 0$ as $n$ goes to infinity. So, $\operatorname{Tr}\left(A^{n}\right)$ is bounded in $R^{+}$. Recalling (Lieb-Simon [3]) that minimizing sequences are bounded in $H^{1}\left(R^{3}\right)^{N}$, we deduce (extracting a 
subsequence if necessary) that

$$
\begin{aligned}
\varphi^{n}{ }_{i} & \rightarrow \varphi_{i}(\text { weak }) \text { in } H^{1}\left(R^{3}\right), \\
A^{n} & \rightarrow A \text { in } L\left(R^{N}\right),
\end{aligned}
$$

and $\varepsilon$, the smallest eigenvalue of $A$, satisfies $\varepsilon>0$.

Now, we can pass to the limit in (6) and we deduce

$$
-\Delta \varphi_{i}+V \varphi_{i}+(\rho * 1 /|x|) \varphi_{i}-\int_{R^{3}} \rho(x, y) \varphi_{i} /|x-y| d x d y+\left(A \varphi, e_{i}\right)+\delta_{1 i} \sum_{l=1, L} \alpha_{l} \underline{\theta}_{l}=0 .
$$

The $\alpha_{l}$ are the limits of the sequence $\left(\alpha_{l}^{n}\right)$, which is bounded in $R$ for each $l$. To see this, we have just to multiply (6) by $\underline{\theta}_{l}$ and integrate: the result follows immediately. On the other hand, multiply again (6) by $\varphi^{n}{ }_{i}$, sum on $i$, and pass to the limit to obtain the identity (the convergence in (6) is strong)

$$
\begin{aligned}
\lim \sup _{n} \int_{R^{3}}\left(A^{n} \varphi^{n}, \varphi^{n}\right) d x= & -\left\{\liminf _{n} \int_{R^{3}} \sum_{i}\left|\nabla \varphi^{n}{ }_{i}\right|^{2}+V\left|\varphi^{n}{ }_{i}\right|^{2}\right) \\
& \left.+\iint_{R^{3} \times R^{3}}\left(\rho^{n}(x) \rho^{n}(y)-\left|\rho^{n}(x, y)\right|^{2}\right) /|x-y| d x d y\right\} .
\end{aligned}
$$

By the same arguments as the ones used to show that $\mathbb{E}$ is weakly lower semicontinuous on $H^{1}\left(R^{3}\right)$ (see Lieb-Simon [3]), this yields

$$
\begin{aligned}
\lim \sup _{n} \int_{R^{3}}\left(A^{n} \varphi^{n}, \varphi^{n}\right) d x \leqq & -\left\{\int_{R^{3}} \sum_{i}\left(\left|\nabla \varphi_{i}\right|^{2}+V\left|\varphi_{i}\right|^{2}\right)+\iint_{R^{3} \times R^{3}}(\rho(x) \rho(y)\right. \\
& \left.\left.-|\rho(x, y)|^{2}\right) /|x-y| d x d y\right\}=\int_{R^{3}}(A \varphi, \varphi) d x .
\end{aligned}
$$

Hence, $\varphi^{n}{ }_{i}$ converges strongly in $L^{2}\left(R^{3}\right)$ to $\varphi_{i}$, and it is easy to conclude the proof of our theorem.

If $Z=N$, we just have to modify slightly the above argument by passing first to the limit weakly in $H^{1}\left(R^{3}\right)$. Then the operator $H=-\Delta+V+(\rho * 1 /|x|)$ still has at most $N+K$ eigenvalues less or equal than $-\varepsilon, \varepsilon$ defined as below. Now if $\int_{R^{3}} \rho=N$ this means that $\varphi^{n}{ }_{i}$ converges strongly in $L^{2}\left(R^{3}\right)$ to $\varphi_{i}$, and the proof is over. Otherwise, we apply Lemma 1 to show that $\varepsilon>0$; this enables us to conclude as before.

Appendix. We rewrite the proof of Lemma 1 from Lions [4].

Lemma 1. Let $\mu$ be a bounded non-negative measure on $R^{3}$ such that $\mu\left(R^{3}\right)<Z$. Let $H_{1}$ be the Hamiltonian given by

$$
H_{1}=-\Delta+V+\mu * 1 /|x| \text {. }
$$

Then $H_{1}$, admits an increasing sequence of negative eigenvalues $\lambda_{n}$ converging to 0 as $n$ goes to $\infty$.

Proof. It is enough to find for each integer $k$ a subspace of dimension $k$ which 
we denote by $F_{k}$, such that $\operatorname{Min}\left\{\left(H_{1} \varphi, \varphi\right) / \varphi \in F_{k},\|\varphi\|_{2}=1\right\}<0$. To find such a $F_{k}$, we consider an arbitrary normalized function

$$
\begin{aligned}
& \varphi \in D\left(R^{3}\right) \text { and we set } \\
& \varphi_{\sigma}(x)=\sigma^{-3 / 2} \varphi(x / \sigma) \text { for } \sigma>0 .
\end{aligned}
$$

Then we have

$$
\left(H_{1} \varphi_{\sigma}, \varphi_{\sigma}\right)=\sigma^{-2}\|\operatorname{grad} \varphi\|_{2}^{2}+\sigma^{-1} \int_{R^{3}}\left(V_{\sigma}(x)|\varphi|^{2}\right) d x+\sigma^{-1} \int_{R^{3}}\left(\mu_{\sigma} * 1 /|x|\right)|\varphi|^{2} d x,
$$

where $V_{\sigma}(x)=-\sum_{j=1, m} z_{j} /\left|x-\left(x_{j} / \sigma\right)\right|$ and $\mu_{\sigma}=\sigma^{3} \mu\left(\sigma_{*}\right)$. In particular, if we choose $\varphi$ to be radially symmetric, we may write the last term as

$$
\begin{aligned}
\int_{R^{3}}\left(\mu_{\sigma} * 1 /|x|\right)|\varphi|^{2} d x & =\int_{R^{3}}\left(|\varphi|^{2} * 1 /|x|\right) d \mu_{\sigma} \\
& =\iint_{R^{3} \times R^{3}}|\varphi(y)|^{2} \max (|x|,|y|)^{-1} d \mu_{\sigma}(x) d y \\
& \leqq \mu_{\sigma}\left(R^{3}\right) \int_{R^{3}}|\varphi(y)|^{2} /|y| d y
\end{aligned}
$$

Now choosing any $k$-dimensional space of radially symmetric functions in $D\left(R^{3}\right)$ and denoting $F_{k}$ the space obtained by rescaling them $\left(\varphi \rightarrow \varphi_{\sigma}\right)$ as above, we obtain the result for $\sigma$ large enough.

Acknowledgements. I wish to thank P. L. Lions for valuable discussions on the topics of this paper, the referee for his suggestions and L. C. Evans for polishing the English of the final version of this paper.

\section{References}

1. Cowan, C.: The theory of atomic structure and spectra. Universes of California Press 1981

2. Ekeland, I.: Non-convex minimization problems. Bull. Am. Math. Soc. 1, 443-474 (1979)

3. Lieb, E., Simon, B.: The Hartree-Fock theory for Coulomb systems. Commun. Math. Phys. 53, 185194 (1977)

4. Lions, P. L.: Solution of Hartree-Fock equations for Coulomb systems. Commun. Math. Phys. 109, 33-97 (1987)

Communicated by B. Simon

Received July 2, 1987; in revised form June 28, 1988 